\title{
ACCELERATION PHENOMENA OBSERVED BY INTERPLANETARY SCINTILLATION AT 0.1-0.3 AU
}

\author{
M. KOJIMA, H. MISAWA, H. WATANABE and Y. YAMAUCHI \\ Solar-Terrestrial Environment Laboratory, Nagoya University, Toyokawa 442, Japan
}

\begin{abstract}
.
Interplanetary scintillation has observed high-latitude streams which show low speeds $(\leq 400 \mathrm{~km} / \mathrm{s})$ within $0.3 \mathrm{AU}$ and high speeds $(\geq 700 \mathrm{~km} / \mathrm{s})$ beyond $0.3 \mathrm{AU}$. The foot points of these streams were located in the HeI coronal holes or at their boundaries. Since the IPS measurement is biased by one or several mechanisms, we investigated whether this acceleration phenomena could be caused by bias effects or not. Speed increase of more than $100 \mathrm{~km} / \mathrm{s}$ is left without being explained by the bias effects.
\end{abstract}

Key words: Interplanetary scintillation - solar wind acceleration - turbulence level within $0.3 \mathrm{AU}$

\section{Introduction}

The behavior of the solar wind as a function of radial distance from the Sun has been studied at distances of $0.3-1 \mathrm{AU}$ with spacecraft and IPS. Intriligator and Neugebauer (1975) reported no statistically significant changes of the average velocity in the region 0.7 and $1 \mathrm{AU}$ from analysis of Pioneer 9 observations. Coles and Rickett (1976) reported from the IPS observations near the ecliptic plane that there is no significant variation of the average solar wind speed between 0.4 and 1.1 AU. Analyzing Helios I and Helios II data Schwenn et al. (1981) found that the radial gradient of the flow speed differs significantly in high and low speed winds. The speed of the slow solar wind increased by $(52 \pm 11) \mathrm{km} / \mathrm{s} / \mathrm{AU}$ over the distance range of $0.3-1 \mathrm{AU}$, while the fast streams remained nearly constant. The solar wind within $0.3 \mathrm{AU}$ has not been observed directly by spacecraft. However, measurements in these regions are extremely important to distinguish between possible acceleration mechanisms. The IPS method has been used to study the outflow properties of the solar wind plasma in these regions (e.g. Armstrong et al., 1986; Armstrong et al., 1990; Bird et al., 1991; Bourgois and Coles, 1991; Coles et al., 1991; Kojima et al., 1991; Tokumaru et al., 1991; Misawa and Kojima, 1992; Bird et al., 1994; Woo and Goldstein, 1994). Kojima et al. (1991) showed that the large scale structure of speed distribution in the longitude and latitude coordinates differs significantly within and beyond $0.3 \mathrm{AU}$. In order to clarify this phenomenon, measurements have to be made at different heliocentric distances in the same flow tube over short periods of time in order to distinguish changes in the solar wind in time or space. Coles et al. (1991) made such systematic measurements using the 
EISCAT facility in Nothern Scandinavia, which covered the distance range from 20 to 120 Rs. Low-speed and medium-speed streams were observed in their work and about half of the radial distance dependencies of a speed could be explained with theoretical solar wind models.

For a spherical symmetric solar wind, the usual assumption is that the IPS measures the solar wind around the closest point "P" to the Sun on a line-of-sight. This "P-point assumption" has been shown to be valid in most observations beyond $0.5 \mathrm{AU}$, especially for the low speed wind, by comparing speeds measured by spacecraft and those measured by IPS (Watanabe and Kakinuma, 1972; Coles et al., 1978; Coles and Kaufman, 1978). The flow speeds deduced from IPS measurements are actually an integral of the speed transverse to the line-of-sight weighted by the density variance. In the following we will therefore use the expression "IPS speed", "P-latitude", and "P-distance". The biases in the IPS speed measurement have been discussed analytically for unstructured spherically symmetric solar wind by Jokipii and Lee (1973). For the structured solar wind, however, it is difficult to estimate the bias because the integration effect depends on the threedimensional distribution of the solar wind properties (the turbulence level of electron density and speed) and the geometry of the line-of-sight through the solar wind. In the following sections we examine some of the possible bias effects in the structured solar wind.

\section{Radial distance dependence of IPS speed}

Regular IPS observations have been performed at a frequency of $327 \mathrm{MHz}$ by the Solar-Terrestrial Environment Laboratory since the early 1980s (kojima et al., 1982; Misawa et al., 1991). The IPS observation at this frequency can cover the distance range from 0.1 to 1 AU. Kojima and Kakinuma (1990) analyzed the radial distance dependence of the IPS speed and found that both of a low-speed wind and a high-speed wind show large speed increase at distance range of 0.1-0.3 AU and become stable flow beyond 0.3 AU (Figure 1). Misawa and Kojima (1992) and Kojima et al. (1995) investigated when in solar activity phase many of the streams with the large speed increase tend to be observed and where they have source regions on the Sun. Major of them were observed in the minimum phase of solar activity and at high latitudes from the magnetic neutral line. They also found that the stream foot points tend to distribute in HeI polar coronal holes and their boundaries. However it should be noted that in these analysis the so called "P-point assumption" were used. Therefore the distances are of the closest point on the line-of-sight to the Sun. 


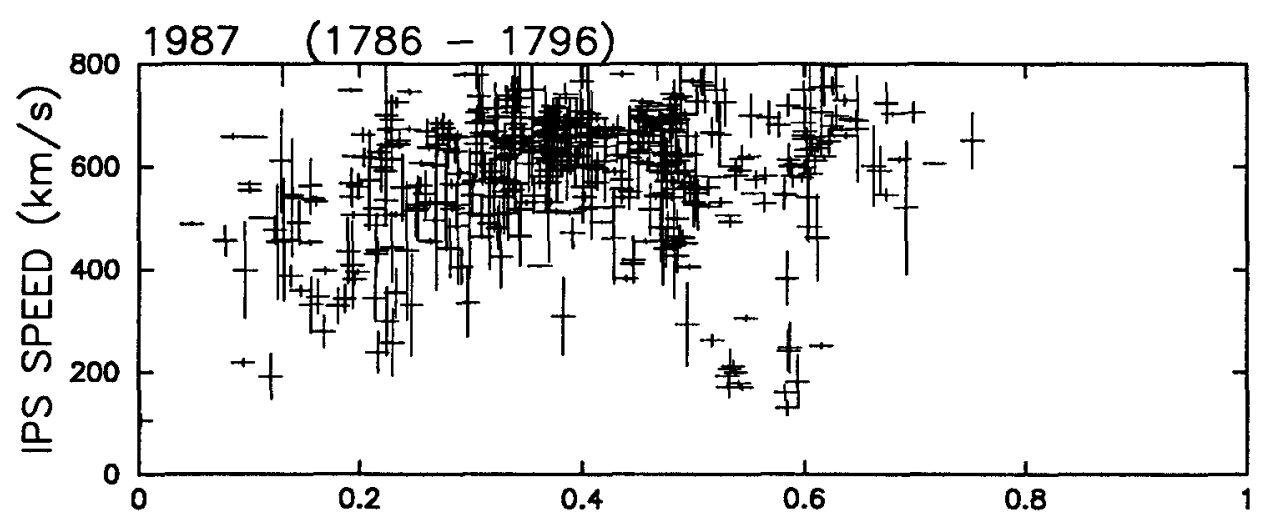

HELIOCENTRIC DISTANCE (AU)

Fig. 1. Distance dependence of the IPS speed observed in high-speed regions. The high-speed regions are defined as the longitude and latitude regions in a v-map where the IPS speed is faster than $600 \mathrm{~km} / \mathrm{s}$ at the distances of $0.3-1 \mathrm{AU}$. All data obtained during the indicated period in 1987 are plotted.

\section{Biases in interplanetary scintillation measurements}

Since the IPS method is an indirect measurement, the IPS speed is biased by one or several mechanisms. The bias effects are different at different radial distances from the Sun and for different 3-dimensional structures of the solar wind. We will examine whether the acceleration phenomena observed within $0.3 \mathrm{AU}$ at high latitudes could be caused by bias effects by simulating the IPS observations with the Born approximation (Kakinuma et al., 1982).

We use the latitudinal structures of the speed and turbulence level of density shown in Figure 2a for the IPS simulation. Here the solar wind speed is taken to be constant along the radial direction, while the turbulence level is assumed to vary with radial distance and velocity according to $R^{-2} V^{-\alpha}$. If the turbulence level, $\delta N_{e}$, is linearly proportional to density, $N_{e}, \alpha=1$ means $N_{e} V=$ constant and $\alpha=2$ means $N_{e} V^{2}=$ constant. Figure $2 \mathrm{~b}$ and 3 show the IPS speeds calculated for various latitudinal structures. The abscissa of Figures is the latitude of the boundary between high-speed and low-speed regions. The IPS speeds in Figure $2 \mathrm{~b}$ are calculated at the elongation angles of $10^{\circ}$ when the line-of-sight is in the meridian plane, while Figure 3 shows the IPS speed at three different elongation angles of $10^{\circ}$ (circles), $20^{\circ}$ (squares) and $30^{\circ}$ (triangles). The distance of the closest approach to the Sun on a line-of-sight (P-distance) is $0.2,0.3$ and $0.5 \mathrm{AU}$ at each elongation, and the corresponding latitudes (P-latitude) are $80^{\circ}$, 

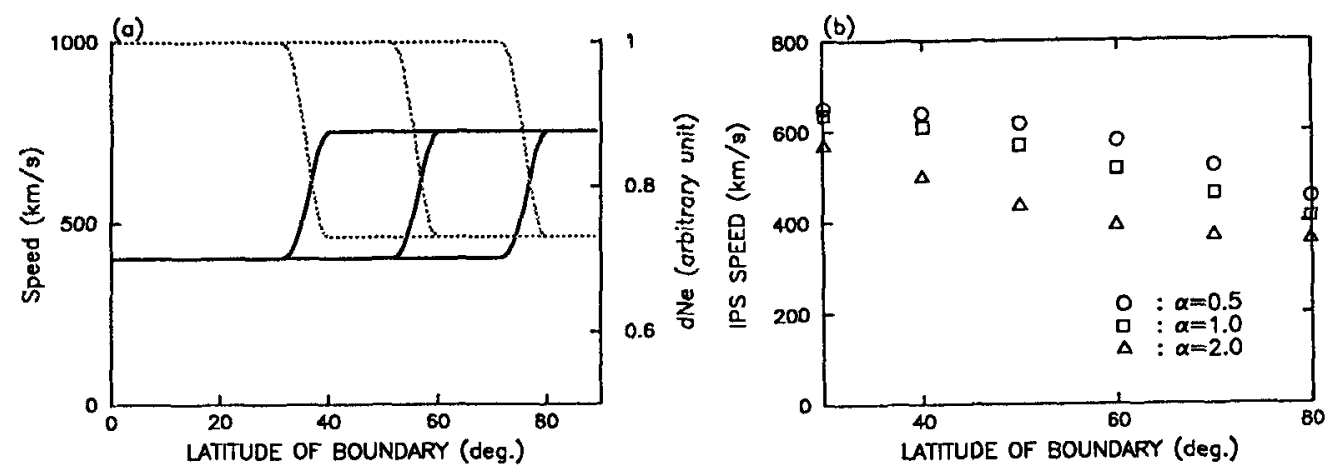

Fig. 2. (a) Model of the latitudinal structure of the speed (solid lines) and the turbulence level (dotted lines). The speed is $750 \mathrm{~km} / \mathrm{s}$ at high latitudes and $400 \mathrm{~km} / \mathrm{s}$ at low latitudes. The turbulence level, $\delta N_{e}$, is assumed to be $\delta N_{e} \propto R^{-2} V^{-\alpha}$ and the example of $\alpha=0.5$ is demonstrated. (b) IPS speeds calculated in the model solar wind shown in Figure 2a. A line-of-sight is in the meridian plane with the elongation angle of $10^{\circ}$. The speed is shown as a function of the latitude representing a boundary between low-speed and high-speed regions.

$70^{\circ}$ and $60^{\circ}$, respectively. The power of the speed, which represents the turbulence level, is assumed to be $\alpha=0.5$ in Figure $3 \mathrm{a}$ and $\alpha=2.0$ in Figure 3b.

Figure 3 shows that the IPS speed is biased toward lower at a larger elongation angle, that is, the IPS speed observed within $0.3 \mathrm{AU}$ should be larger than that observed beyond $0.3 \mathrm{AU}$. We also simulated IPS observations for the radio sources which were observed during April and July in 1985 with the model solar wind shown in Figure 2 in the paper of Kojima and Kakinuma (1990). This model is a good representative of the solar wind structure in the minimum phase of the solar activity; a low speed region distributes parallel to the equator with a latitudinal width of $30^{\circ}$. The simulated IPS speed are plotted with respect to a heliocentric distance in Figure 4.The data which are observed at P-latitude larger than $40^{\circ}$ are shown. This Figure also shows that the IPS speed increases as approaching the Sun rather than decreases. These results of the simulation can be explained by the behavior of a weighting function which becomes sharper as approaching to the Sun.

\section{Distance dependence of turbulence level}

Coles et al. (1995) estimated the level of turbulence at solar distances of 5 to 15 Rs from coherent scintillation bandwidth observations and found that in solar minimum phase $\delta N_{e}^{2}$ in the polar fast stream is a factor of 15 smaller 

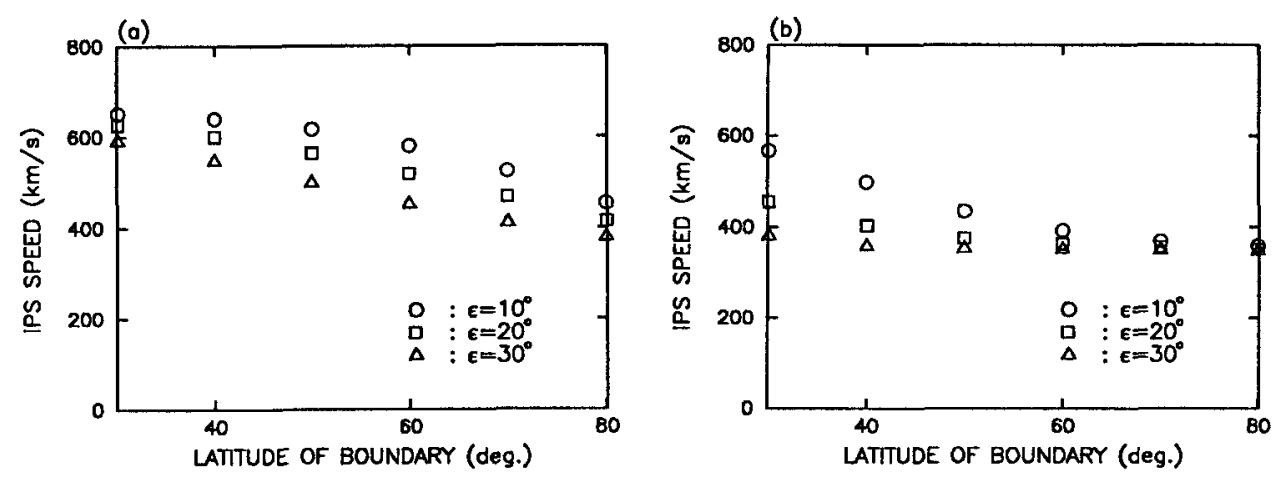

Fig. 3. IPS speeds calculated for the examples of (a) $\alpha=0.5$ and (b) $\alpha=2$ in the model solar wind shown in Figure 2a. The IPS speeds were calculated at three different elongation angles of $10^{\circ}, 20^{\circ}$ and $30^{\circ}$.

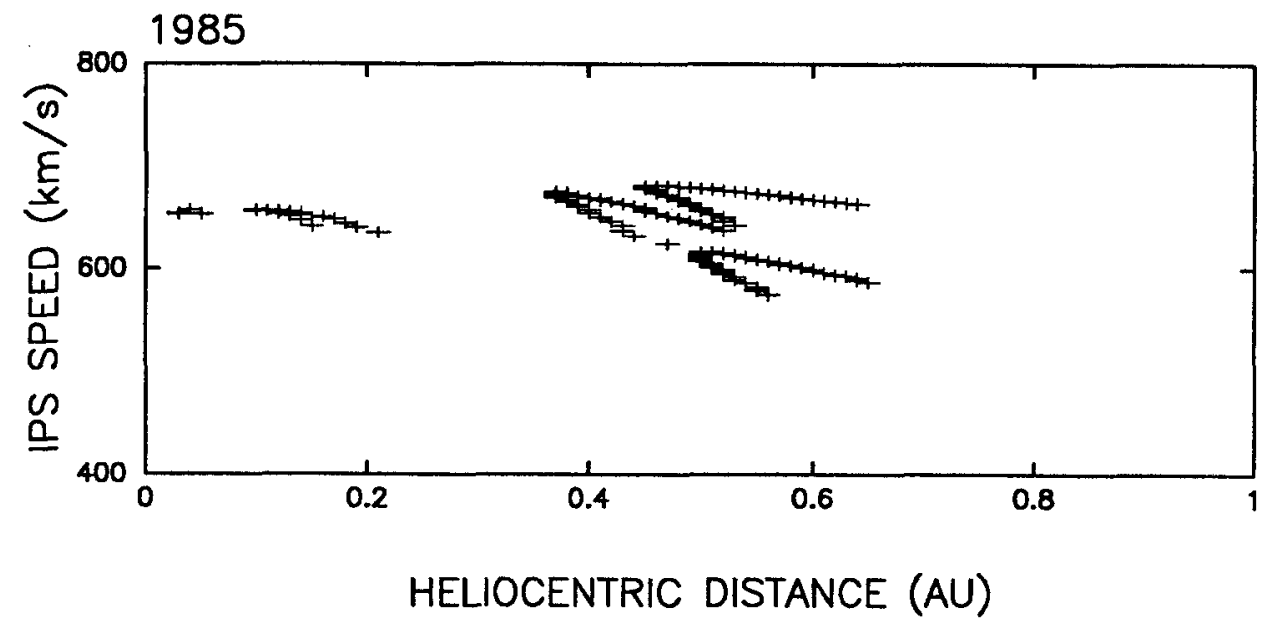

Fig. 4. Distance dependence of the IPS speed simulated for the model solar wind shown in Figure 2 in the paper of Kojima and Kakinuma (1990). The data simulated at P-latitudes larger than $40^{\circ}$ are plotted.

solar minimum phase $\delta N_{e}^{2}$ in the polar fast stream is a factor of 15 smaller than that in the lower latitude wind. This factor of 15 corresponds to $\alpha \simeq 2.2$ in our model. Manoharan (1993) estimated the latitudinal dependence of $\delta N_{e}$ in the solar minimum phase from scintillation index measurements. He reported that $\delta N_{e}$ at a distance of $45 \mathrm{Rs}$ is a factor of 2.5 smaller at a high latitude than that at a low latitude. The factor of 2.5 for $\delta N_{e}$ corresponds 
has a smaller angular width than $40^{\circ}$ is hidden from the IPS observations because of the low turbulence level. This can be seen in Figure $2 \mathrm{~b}$ and $3 \mathrm{~b}$ : when $\alpha=2.0$, the IPS does not observe a high speed $(\geq 500 \mathrm{~km} / \mathrm{s})$ at elongations larger than $20^{\circ}$ even if a $750 \mathrm{~km} / \mathrm{s}$ high-speed region has large latitudinal extension of $60^{\circ}$ from the pole. However IPS radio sources at southern high latitudes such as 3C161 observed IPS speeds of around $600 \mathrm{~km} / \mathrm{s}$ in 1994 when the Ulysses observed stable high speeds around $750 \mathrm{~km} / \mathrm{s}$ at high latitudes. This observational evidence indicates that the density turbulence in a high-speed stream beyond $0.3 \mathrm{AU}$ is not so small as that found within $15 \mathrm{Rs}$ by Coles et al. (1995). Since the Ulysses observed the latitudinal boundary at $40^{\circ}, \alpha$ should be 0.5 to explain the IPS observations of $600 \mathrm{~km} / \mathrm{s}$. Kakinuma et al. (1982) showed that $\alpha=0.5$ can well explain the IPS speeds observed beyond 0.3 AU.

The discrepancy between the estimated $\delta N_{e}$ within $15 \mathrm{Rs}$ by Coles et al. (1995), at 45 Rs by Manoharan (1993) and at $\mathrm{R} \geq 60$ Rs by Kakinuma et al. (1982) may indicate the evolution of the turbulence level in a high-speed wind. In this case, relative contribution to the bias from a low-speed region increases as approaching the Sun, and consequently the IPS speed will be decreased by the bias. We can see this in comparison of Figures $3 \mathrm{a}$ and $3 \mathrm{~b}$. When the latitude of boundary is $40^{\circ}$, the IPS speed is about $550 \mathrm{~km} / \mathrm{s}$ at the P-distance of about $100 \mathrm{Rs}$ for $\alpha=0.5$ while it is $500 \mathrm{~km} / \mathrm{s}$ at about $40 \mathrm{Rs}$ for $\alpha=2$. Thus difference of the IPS speeds at $40 \mathrm{Rs}$ and $100 \mathrm{Rs}$ is about $50 \mathrm{~km} / \mathrm{s}$. Even if the latitude of boundary changes from $30^{\circ}$ to $80^{\circ}$, the difference does not change. Consequently variation of the turbulence level with a heliocentric distance cannot explain the acceleration phenomena.

\section{Bias in strong scattering}

In the previous sections, IPS speeds were calculated for model solar winds using the Born approximation which is valid in the weak scattering region. Since scattering at $327 \mathrm{MHz}$ is strong at distances within about $0.2 \mathrm{AU}$, biases in a strong scattering region have to be examined. Kojima et al. (1995) examined strong scattering effects on the IPS speed measurements using the IPS data measured at frequencies of $69 \mathrm{MHz}$ and $74 \mathrm{MHz}(\mathrm{VHF})$ by the STE Lab. and UCSD. At these radio frequencies strong scattering begins at distances $\leq 100$ Rs. To see the strong scattering effect, the IPS data obtained within the heliographic latitude band of $\pm 10^{\circ}$ during the Carrington rotations of 1640 to 1645 in 1976 were plotted as the function of a distance. The IPS speeds observed at VHF do not show decrease when passing into the strong scattering region, and this is consistent with the Helios observations which show rather constant speed between 0.3 and 1 
AU (Schwenn et al., 1981). This means that the strong scattering effect does not seem to bias the IPS speeds to lower values.

\section{Discussion}

Through the whole solar activity cycle the IPS has observed high-latitude solar wind streams which have large differences in the derived IPS speeds between the distance ranges both within and beyond $0.3 \mathrm{AU}$. The foot points of these streams were located in HeI coronal holes or at their boundaries. We have investigated several mechanisms which could lead to biasing the derived IPS speeds towards lower values when the IPS observations are made closer to the Sun.

IPS observations were simulated assuming the turbulence level of the form $\delta N_{c}=V^{-\alpha} R^{-2}$. This simulation shows that if the turbulence level is constant at all distance range, the IPS speed does not decrease as the P-distance approaches to the Sun. The simulations also found that if $\alpha=2$, the observed IPS speed will not be larger than $500 \mathrm{~km} / \mathrm{s}$ at larger than $20^{\circ}$ elongation angles. However there is evidence that the IPS observes high speeds ( $\geq 600 \mathrm{~km} / \mathrm{s}$ ) at large elongations. Therefore $\alpha$ is probably not as large as 2 beyond $0.3 \mathrm{AU}$, that is, $\delta N_{e}$ for $\mathrm{R} \geq 0.3 \mathrm{AU}$ is not as small as those measured within $45 \mathrm{Rs}$ by Coles et al. (1995) and Manoharan (1993). We then examined how much the IPS speed can be reduced if the turbulence level decreases within $0.3 \mathrm{AU}$. For this analysis we employed a model that the turbulence level changes with a distance: $\alpha=0.5$ beyond $0.3 \mathrm{AU}$ and $\alpha=2$ within $0.3 \mathrm{AU}$. This examination shows that if $\delta N_{e}=V^{-0.5}$ or $V^{-1}$ are assumed, it is difficult to reduce the IPS speed more than $50 \mathrm{~km} / \mathrm{s}$ by changing only the radial distance dependence of $\delta N_{\epsilon}$.

Finally we have discussed the strong scattering effect since the IPS observations within $0.2 \mathrm{AU}$ are in the strong scattering region at a frequency of $327 \mathrm{MHz}$. However, no evidence showing the decrease of the IPS speed in the strong scattering region was found.

Speed differences of $100 \mathrm{~km} / \mathrm{s}$ between within and beyond $0.3 \mathrm{AU}$ can be explained by present acceleration models (eg., Parker, 1965; Coles et al., 1991; Barnes, 1992). The differences of another $50 \mathrm{~km} / \mathrm{s}$ can be explained by the bias effects when the change of the turbulence level with a distance exists. However the difference more than $100 \mathrm{~km} / \mathrm{s}$ is left without being explained. If it were caused by some bias effects which were not considered in this paper, the biases have to work only in the observations within $0.3 \mathrm{AU}$. We employed a simple solar wind model to simulate the IPS observations. If the bias were caused by transverse of a line-of-sight through more complicated structure in the solar wind, the bias effects should appear at all distance range but not only within $0.3 \mathrm{AU}$. According to these considerations we are 
convinced that the acceleration phenomena observed at $0.1-0.3 \mathrm{AU}$ by the IPS method are not apparent but real.

\section{Acknowledgements}

We especially thank Wm. A. Coles, UCSD, for providing the IPS data observed with the VHF antenna system of UCSD and for his valuable comments and discussion on this work. We wish to thank R. Esser for her critical and valuable remarks about this work. We would like to acknowledge engineering supports by Y. Ishida, K. Maruyama and N. Yoshimi, and to thank M. Yoshida for her assistance in data analysis. This research was supported by the Scientific Research Fund of the Ministry of Education, Culture, and Science (grant 04640424).

\section{References}

Armstrong, J. W., W.A. Coles, M. Kojima and B.J. Rickett: 1986, The Sun and Heliosphere in Three-Dimensions, D. Reidel Publishing Company: North Holland, 59.

Armstrong, J. W., W.A. Coles, M. Kojima and B.J. Rickett: 1990, Ap. J. 358, 685.

Barnes, A.: 1992, Rev. Geophys. 30, 43.

Bird, M. K., H. Volland, A.I. Efimov, G.S Levy, B.L. Seidel and C.T. Stelzried: 1991, Solar Wind Seven, Pergamon Press: Oxford, 147.

Bird, M. K., H. Volland, M. Patzold, P. Edenhofer, S.W. Asmar and J.P. Brenkle: 1994, Ap. J. 426, 373 .

Bourgois, G., and W.A.Coles: 1991, Solar Wind Seven, Pergamon Press: Oxford, 155.

Coles, W.A., J. K. Harmon, A.J. Lazarus, and J.D. Sullivan: 1978, J. Geophys. Res. 83, 3337.

Coles, W. A., and J. J. Kaufman: 1978, Radio Science 13, 591.

Coles, W.A., R.R. Grall, M.T. Klinglesmith, and G. Bourgois: 1995, private communication.

Coles, W. A., and B. J. Rickett: 1976, J. Geophys. Res. 81, 4797.

Coles, W. A., R. Esser, U.-P. Lovhaug and J. Markkanen: 1991, J. Geophys. Res. 96, 13849.

Intriligator, D. S., and M. Neugebauer: 1975, J. Geophys. Res. 80, 1332.

Jokipii, J. R., and L.C. Lee: 1973, Ap. J. 182, 317.

Kakinuma, T., H. Washimi and M. Kojima: 1982, Proc. STIP Symp. on Solar Radio Astronomy, Interplanetary scintillation and coordination with spacecraft, AFGL Special Report \#233, 153.

Kojima, M., Y. Ishida, K. Maruyama and T. Kakinuma: 1982, Proc. Res. Inst. Atmospherics, Nagoya Univ. 29, 61.

Kojima, M., and T. Kakinuma: 1990, Space Science Rev. 53, 173.

Kojima, M., H. Washimi, H. Misawa and K. Hakamada: 1991, Solar Wind Seven, Pergamon Press: Oxforded, 201.

Kojima, M., K. Asai, Y. Kozuka, H. Misawa, H. Watanabe and Y. Yamauchi: 1995, Adv. Space Res., Pergamon Press: Oxforded, 16, 101.

Manoharan, P.K.: 1993, Solar Physics 148, 153.

Misawa, H., Y. Ishida, M. Kojima, K. Maruyama and N. Yoshimi: 1991, Proc. of the Second STEP Symposium, 86.

Misawa, H., and M. Kojima: 1992, Proc. of the Third STEP Symposium, 89. 
Parker, E. N.:1965, Space Sci. Rev. 4, 666.

Schwenn. R. M., K.-H. Muhlhauser, E. Marsch and H. Rosenbauer: 1981, Solar Wind Four, ed. H. Rosenbauer, Max-Planck-Institute fur Aeronomy, Katlenburg-Lindau and MaxPlanck-Institute fur Extraterrestrische Physic, Garching, West Germany, 126.

Tokumaru, M., H. Mori, T. Tanaka, T. Kondo, H. Takaba and Y. Koyama: 1991, J. Geomag. Geoelectr 43, 619.

Watanabe, T. and T. Kakinuma: 1972, Publ. Astron. Soc. Japan. 24, 459.

Woo, R., and R.M. Goldstein: 1994, Geophys. Res. Let. 2185. 